\title{
New approach to conserved charges of generic gravity in AdS spacetimes
}

\author{
Emel Altas ${ }^{*}$ \\ Department of Physics, Karamanoglu Mehmetbey University, 70100 Karaman, Turkey \\ Bayram Tekin ${ }^{\dagger}$ \\ Department of Physics, Middle East Technical University, 06800 Ankara, Turkey
}

(Received 29 November 2018; published 12 February 2019)

\begin{abstract}
Starting from a divergence-free rank-4 tensor of which the trace is the cosmological Einstein tensor, we give a construction of conserved charges in Einstein's gravity and its higher derivative extensions for asymptotically anti-de Sitter spacetimes. The current yielding the charge is explicitly gauge invariant, and the charge expression involves the linearized Riemann tensor at the boundary. Hence, to compute the mass and angular momenta in these spacetimes, one just needs to compute the linearized Riemann tensor. We give two examples.
\end{abstract}

DOI: $10.1103 /$ PhysRevD.99.044016

\section{INTRODUCTION}

Let us start with a seemingly innocent question which will have far-reaching consequences for the conserved charges of gravity theories. Given the Riemann tensor $R_{\mu \beta \sigma}^{\nu}$, its single trace (over the first and third indices) yields the Ricci tensor $R_{\mu \sigma}$; is there a rank-4 tensor of which the single trace is not the Ricci tensor but the (cosmological) Einstein tensor, $\mathcal{G}_{\mu \sigma}=R_{\mu \sigma}-\frac{1}{2} R g_{\mu \sigma}+\Lambda g_{\mu \sigma}$, with the condition that this four-index tensor has the symmetries of the Riemann tensor and it is divergence free just like the Einstein tensor? Remarkably, the answer is affirmative : the tensor

$$
\begin{aligned}
\mathcal{P}^{\nu \mu \beta \sigma}:= & R^{\nu \mu \beta \sigma}+g^{\sigma \nu} R^{\beta \mu}-g^{\beta \nu} R^{\sigma \mu}+g^{\beta \mu} R^{\sigma \nu}-g^{\sigma \mu} R^{\beta \nu} \\
& +\left(\frac{R}{2}-\frac{\Lambda(n-3))}{n-1}\right)\left(g^{\beta \nu} g^{\sigma \mu}-g^{\sigma \nu} g^{\beta \mu}\right)
\end{aligned}
$$

whose construction will be given below does the job. Its divergence-free for all smooth metrics, i.e., without the use of any field equations

$$
\nabla_{\nu} \mathcal{P}_{\mu \beta \sigma}^{\nu}=0
$$

and its trace is the cosmological Einstein tensor as desired,

$$
\mathcal{P}^{\nu}{ }_{\mu \nu \sigma}=(3-n) \mathcal{G}_{\mu \sigma} .
$$

*emelaltas@kmu.edu.tr

btekin@metu.edu.tr

Published by the American Physical Society under the terms of the Creative Commons Attribution 4.0 International license. Further distribution of this work must maintain attribution to the author(s) and the published article's title, journal citation, and DOI. Funded by SCOAP ${ }^{3}$.
Clearly, the interesting exception is that one cannot do this construction in three dimensions. What happens for $n=3$ is that the $\mathcal{P}$-tensor vanishes identically since, due to the vanishing of the Weyl tensor, the Riemann and the Ricci tensors carry the same amount of information and the Riemann tensor can be expressed in terms of the Ricci tensor as

$$
\begin{aligned}
R^{\nu \mu \beta \sigma}= & R^{\nu \beta} g^{\mu \sigma}+R^{\mu \sigma} g^{\nu \beta}-R^{\nu \sigma} g^{\mu \beta}-R^{\mu \beta} g^{\nu \sigma} \\
& -\frac{R}{2}\left(g^{\nu \beta} g^{\mu \sigma}-g^{\mu \beta} g^{\nu \sigma}\right) .
\end{aligned}
$$

Therefore, in some sense, the $\mathcal{P}$-tensor (1) is an obstruction for a smooth generically curved metric to be three dimensional. This can also be seen from the following identity: the Gauss-Bonnet combination $\chi_{\mathrm{GB}}:=R^{\nu \mu \beta \sigma} R_{\nu \mu \beta \sigma}-4 R_{\mu \nu} R^{\mu \nu}+R^{2}$ vanishes identically in three dimensions, and it is easy to show that the contraction of the $\mathcal{P}$-tensor with the Riemann tensor yields ${ }^{1}$

$$
R^{\nu \mu \beta \sigma} \mathcal{P}_{\nu \mu \beta \sigma}=\chi_{\mathrm{GB}}-2 \Lambda \frac{(n-3)}{n-1} R
$$

\footnotetext{
${ }^{1}$ On a curious note, one can see that the square of this tensor yields a particular Einstein plus quadratic gravity in generic $n \geq 4$ dimensions,

$$
\begin{aligned}
\mathcal{P}_{\nu \mu \beta \sigma}^{2}= & \chi_{\mathrm{GB}}+(n-3)\left(4 R_{\mu \nu}^{2}+\frac{R^{2}}{2}(n-6)\right. \\
& \left.-2 \Lambda \frac{(n-3)}{n-1}((n-2) R-\Lambda n)\right),
\end{aligned}
$$
}

which is not the Lagrangian of critical gravity $[1,2]$. 
which vanishes in three dimensions, but gives the EinsteinGauss-Bonnet Lagrangian (with a fixed relative coefficient) in generic $n$ dimensions. The natural question is how one arrives at the $\mathcal{P}$-tensor (1). We have found the $\mathcal{P}$-tensor from the following construction: starting from the Bianchi identity

$$
\nabla_{\nu} R_{\sigma \beta \mu \rho}+\nabla_{\sigma} R_{\beta \nu \mu \rho}+\nabla_{\beta} R_{\nu \sigma \mu \rho}=0
$$

and carrying out the $g^{\nu \rho}$ multiplication, one arrives at the $\mathcal{P}^{\nu}{ }_{\mu \beta \sigma}$ as given in (1) after making use of $\nabla_{\mu} \mathcal{G}^{\mu \nu}=0$, $\nabla_{\mu} g_{\alpha \beta}=0$. Note that this still leaves an ambiguity in the $\mathcal{P}$-tensor, since one can add an arbitrary constant times $g^{\mu \sigma} g^{\beta \nu}$, but that part can be fixed by demanding that the $\mathcal{P}$-tensor has the symmetries of the Riemann tensor and also vanishes for constant curvature backgrounds, which we assumed. This tensor turns out to be extremely useful in finding conserved charges of Einstein's gravity for asymptotically AdS spacetimes for $n>3$ dimensions. Recently, in Ref. [3], we gave a brief account of this formulation in Einstein's theory, and in the current work, we shall extend this formulation to quadratic and generic gravity theories.

The main motivation of our construction is the following: outside the localized sources, the properties of gravity are fully encoded in the Riemann tensor. One would naturally expect that the charge expression, which is an integral in the boundary of a spacelike surface, would also involve the Riemann tensor at infinity. But a straightforward computation shows that this is not the case, as we shall revisit in the next section. The existing formulas involve the first derivatives of the metric perturbation. The crux of the matter is that the existing expressions are based on conserved currents which are only gauge invariant up to a boundary term that vanishes. Our formalism remedies this and constructs an explicitly gauge-invariant current and simplifies the charge expressions significantly.

The layout of the paper is as follows. In Sec. II, which is the bulk of the paper, we discuss the conserved Killing charges in generic gravity and give a compact expression that utilizes the $\mathcal{P}$-tensor. In Sec. III, we discuss the gaugeinvariance issue of the conserved currents. In Sec. IV, we study the $n$-dimensional Schwarzschild-AdS spacetime and the AdS soliton. In Ref. [3], we studied the Kerr-AdS solution, and hence we shall not repeat it here.

\section{CONSERVED CHARGES}

Conserved charges of generic gravity theory in asymptotically AdS spacetimes were constructed in Ref. [4] as an extension of the Abbott-Deser charges [5] of the cosmological Einstein theory. The latter is a generalization of the Arnowitt-Deser-Misner (ADM) charges [6] which are valid for asymptotically flat spacetimes. A detailed account of these constructions was recently given in Ref. [7], and for related constructions, see Refs. [8] and [9]. Here, for the sake of completeness, we will briefly summarize the salient parts of this construction. Consider a generic gravity theory defined by the field equations depending on the Riemann tensor $(\mathcal{R})$, its derivatives, and contractions,

$$
\mathcal{E}_{\mu \nu}\left(g, \mathcal{R}, \nabla \mathcal{R}, \mathcal{R}^{2}, \ldots\right)=\kappa \tau_{\mu \nu},
$$

where $\nabla_{\mu} \mathcal{E}^{\mu \nu}=0$ and $\kappa$ is the $n$-dimensional Newton constant while $\tau_{\mu \nu}$ represents a localized conserved source. A nontrivial, partially conserved current arises after one splits the metric as

$$
g_{\mu \nu}=\bar{g}_{\mu \nu}+\kappa h_{\mu \nu},
$$

which yields a splitting of the field equations as

$$
\kappa\left(\mathcal{E}_{\mu \nu}\right)^{(1)}(h)=\kappa \tau_{\mu \nu}-\kappa^{2}\left(\mathcal{E}_{\mu \nu}\right)^{(2)}(h)+\mathcal{O}\left(\kappa^{3}\right),
$$

where we assumed that $\bar{g}$ solves the field equations, $\mathcal{E}_{\mu \nu}(\bar{g})=0$, exactly in the absence of any source $\tau_{\mu \nu}$ and $\left(\mathcal{E}_{\mu \nu}\right)^{(1)}(h):=\left.\frac{d}{d \kappa} \mathcal{E}_{\mu \nu}(\bar{g}+\kappa h)\right|_{\kappa=0}$. Hence, defining $\left(\mathcal{E}_{\mu \nu}\right)^{(1)}:=$ $T_{\mu \nu}$, one has the desired partially conserved current, if the background admits a Killing vector $\bar{\xi}$ :

$$
\mathcal{J}^{\mu}:=\sqrt{-\bar{g}} \bar{\xi}_{\nu}\left(\mathcal{E}^{\mu \nu}\right)^{(1)}
$$

As usual, making use of the Stokes theorem, given a spacelike hypersurface $\bar{\Sigma}$, one has the conserved charge for each background Killing vector

$$
Q(\bar{\xi}):=\int_{\bar{\Sigma}} d^{n-1} y \sqrt{\bar{\gamma}} \bar{n}_{\mu} \bar{\xi}_{\nu}\left(\mathcal{E}^{\mu \nu}\right)^{(1)},
$$

where we assumed the that $\mathcal{J}^{\mu}$ vanishes at spacelike infinity. To proceed further and reduce this integral over $\bar{\Sigma}$ to an integral over the boundary $\partial \bar{\Sigma}$, one must know the field equations and express $\bar{\xi}_{\nu}\left(\mathcal{E}_{\mu \nu}\right)^{(1)}$ as a divergence of an antisymmetric 2-tensor. Recently [3], we have shown that, using the $\mathcal{P}$-tensor of the previous section, one can reformulate this problem in the cosmological Einstein theory in AdS spacetimes without using the explicit form of the linearized cosmological Einstein tensor. This is possible because in Einstein spaces (that are not Ricci flat such as the AdS) one has the nice property that the Killing vector can be derived from an antisymmetric "potential" $\overline{\mathcal{F}}_{\mu \nu}$ as

$$
\bar{\xi}^{\mu}=\bar{\nabla}_{\nu} \overline{\mathcal{F}}^{\nu \mu}
$$

where $\overline{\mathcal{F}}^{\nu \mu}=-\frac{2}{\bar{R}} \bar{\nabla}^{\nu} \bar{\xi}^{\mu}$ with $\bar{R}$ being the constant scalar curvature. Although this result is valid for any Einstein space as a background, for concreteness, we shall work in the AdS background, for which we have 


$$
\begin{aligned}
\bar{R}_{\mu \alpha \nu \beta} & =\frac{2 \Lambda}{(n-2)(n-1)}\left(\bar{g}_{\mu \nu} \bar{g}_{\alpha \beta}-\bar{g}_{\mu \beta} \bar{g}_{\alpha \nu}\right), \\
\bar{R}_{\mu \nu} & =\frac{2 \Lambda}{n-2} \bar{g}_{\mu \nu}, \quad \bar{R}=\frac{2 n \Lambda}{n-2} .
\end{aligned}
$$

To find the conserved charges of a gravity theory defined on an asymptotically AdS spacetime $\mathcal{M}$, let us assume that there is an antisymmetric 2 -form, $\mathcal{F}_{\mu \nu}$, on the manifold. Then, one has the exact equation for any smooth metric

$$
\nabla_{\nu}\left(\mathcal{F}_{\beta \sigma} \mathcal{P}^{\nu \mu \beta \sigma}\right)-\mathcal{P}^{\nu \mu \beta \sigma} \nabla_{\nu} \mathcal{F}_{\beta \sigma}=0
$$

Linearization of (14) about the AdS background yields

$$
\bar{\nabla}_{\nu}\left(\left(\mathcal{P}^{\nu \mu \beta \sigma}\right)^{(1)} \overline{\mathcal{F}}_{\beta \sigma}\right)-\left(\mathcal{P}^{\nu \mu \beta \sigma}\right)^{(1)} \bar{\nabla}_{\nu} \overline{\mathcal{F}}_{\beta \sigma}=0
$$

which is the main equation from which we will read the conserved current.

\section{A. Einstein's theory}

Let us recapitulate the main points of Ref. [3]. Using the following equivalent form of the $\mathcal{P}$-tensor, written in terms of the cosmological Einstein tensor,

$$
\begin{aligned}
\mathcal{P}^{\nu}{ }_{\mu \beta \sigma}:= & R^{\nu}{ }_{\mu \beta \sigma}+\delta_{\sigma}^{\nu} \mathcal{G}_{\beta \mu}-\delta_{\beta}^{\nu} \mathcal{G}_{\sigma \mu}+\mathcal{G}_{\sigma}^{\nu} g_{\beta \mu}-\mathcal{G}_{\beta}^{\nu} g_{\sigma \mu} \\
& +\left(\frac{R}{2}-\frac{\Lambda(n+1)}{n-1}\right)\left(\delta_{\sigma}^{\nu} g_{\beta \mu}-\delta_{\beta}^{\nu} g_{\sigma \mu}\right),
\end{aligned}
$$

one arrives at its linearized form

$$
\begin{aligned}
\left(\mathcal{P}^{\nu \mu \beta \sigma}\right)^{(1)}= & \left(R^{\nu \mu \beta \sigma}\right)^{1}+2\left(\mathcal{G}^{\mu[\beta}\right)^{(1)} \bar{g}^{\sigma] \nu} \\
& +2\left(\mathcal{G}^{\nu[\sigma}\right)^{(1)} \bar{g}^{\beta] \mu}+(R)^{(1)} \bar{g}^{\mu[\beta} \bar{g}^{\sigma] \nu} \\
& +\frac{4 \Lambda}{(n-1)(n-2)}\left(h^{\mu[\sigma} \bar{g}^{\beta] \nu}+\bar{g}^{\mu[\sigma} h^{\beta] \nu}\right),
\end{aligned}
$$

where the square brackets denote antisymmetrization with a factor of $1 / 2$. For the particular antisymmetric background tensor

$$
\overline{\mathcal{F}}_{\alpha \beta}:=\bar{\nabla}_{\alpha} \bar{\xi}_{\beta}
$$

where $\bar{\xi}_{\beta}$ is an AdS Killing vector, one finds from (15) the following conserved current:

$$
\bar{\xi}_{\lambda}\left(\mathcal{G}^{\lambda \mu}\right)^{(1)}=\frac{(n-1)(n-2)}{4 \Lambda(n-3)} \bar{\nabla}_{\nu}\left(\left(\mathcal{P}^{\nu \mu \beta \sigma}\right)^{(1)} \overline{\mathcal{F}}_{\beta \sigma}\right) .
$$

Comparing this with the integrand of (11), and using the Stokes theorem one more time, we find the desired result
$Q(\bar{\xi})=\frac{(n-1)(n-2)}{8(n-3) \Lambda G \Omega_{n-2}} \int_{\partial \bar{\Sigma}} d^{n-2} x \sqrt{\bar{\gamma}} \bar{\epsilon}_{\mu \nu}\left(R_{\beta \sigma}^{\nu \mu}\right)^{(1)} \overline{\mathcal{F}} \beta \sigma$,

where $\left(R_{\beta \sigma}^{\nu \mu}\right)^{(1)}$ is the linearized part of the Riemann tensor about the AdS background. Observe that on the boundary $\left(\mathcal{P}_{\beta \sigma}^{\nu \mu}\right)^{(1)}=\left(R_{\beta \sigma}^{\nu \mu}\right)^{(1)}$, since the linearized Einstein tensor and the linearized scalar curvature vanish. The barred quantities refer to the background spacetime $\overline{\mathcal{M}}$ with the boundary $\partial \overline{\mathcal{M}}$. The Killing vector is $\bar{\xi} \sigma$ from which one defines the antisymmetric tensor as $\overline{\mathcal{F}}^{\beta \sigma}=\bar{\nabla}^{\beta} \bar{\xi}^{\sigma}$. The spatial hypersurface $\bar{\Sigma}$ is not equal to $\partial \overline{\mathcal{M}}$; hence, $\bar{\Sigma}$ can have a boundary of its own, that is $\partial \bar{\Sigma}$. Here, the antisymmetric 2-form $\epsilon$ has components $\bar{\epsilon}_{\mu \nu}:=\frac{1}{2}\left(\bar{n}_{\mu} \bar{\sigma}_{\nu}-\bar{n}_{\nu} \bar{\sigma}_{\mu}\right)$, where $\bar{n}_{\mu}$ is a normal 1-form on $\partial \overline{\mathcal{M}}$ and $\bar{\sigma}_{\nu}$ is the unit normal 1-form on $\partial \bar{\Sigma}$ and $\bar{\gamma}$ is the induced metric on the boundary. This is sufficient for the conserved charges of the cosmological Einstein theory in AdS. But for a generic theory, one must carry out an analogous computation, which is what we do next. But before that, let us note that for the energy of the spacetime, we have $\bar{\xi}=\partial_{t}$, and (20) becomes

$$
\begin{aligned}
E & :=Q\left(\partial_{t}\right) \\
& =\frac{(n-1)(n-2)}{2(n-3) \Lambda G \Omega_{n-2}} \int_{\partial \bar{\Sigma}} d^{n-2} x \sqrt{\bar{\gamma}}\left(R^{r t}{ }_{r t}\right)^{(1)} \bar{\nabla}^{r} \bar{\xi}^{t},
\end{aligned}
$$

where $r$ is the radial coordinate and one takes $r \rightarrow \infty$ at the end of the computation. Similarly, for the angular momentum, one can take the Killing vector $\bar{\xi}^{\mu}=(0, \ldots, 1,0, \ldots, 0)$ and carry out the computation.

\section{B. Generic theory}

Consider a generic gravity theory which starts with the Einsteinian part as

$$
\mathcal{E}_{\mu \nu}=\frac{1}{\kappa}\left(R_{\mu \nu}-\frac{1}{2} R g_{\mu \nu}+\Lambda_{0} g_{\mu \nu}\right)+\sigma E_{\mu \nu}=\tau_{\mu \nu},
$$

where at this stage all we know about the $E_{\mu \nu}$-tensor is that it is a symmetric divergence-free tensor (which can come from an action) and $\sigma$ is a dimensionful parameter. To proceed further, it is better to recast the equation as

$$
\mathcal{E}_{\mu \nu}=\frac{1}{\kappa} \mathcal{G}_{\mu \nu}+\frac{\Lambda_{0}-\Lambda}{\kappa} g_{\mu \nu}+\sigma E_{\mu \nu}=\tau_{\mu \nu}
$$

the $(A) d S$ vacua of which are determined by

$$
\overline{\mathcal{E}}_{\mu \nu}=\frac{\Lambda_{0}-\Lambda}{\kappa} \bar{g}_{\mu \nu}+\sigma \bar{E}_{\mu \nu}=0,
$$

which in general has many vacua depending on the details of the $\bar{E}_{\mu \nu}$ tensor. We shall assume that $\Lambda$ represents any one 
of the viable vacua. To find the conserved charges in this theory, we use the same procedure as the one in the previous section and define

$$
\left(\mathcal{E}_{\mu \nu}\right)^{(1)}=T_{\mu \nu}
$$

where the right-hand side has all the higher order terms

$$
T_{\mu \nu}=\tau_{\mu \nu}-\kappa\left(\mathcal{E}_{\mu \nu}\right)^{(2)}-\kappa^{2}\left(\mathcal{E}_{\mu \nu}\right)^{(3)}-\cdots
$$

So, we have the background conserved current

$$
\bar{\nabla}_{\nu}\left(\bar{\xi}_{\mu}\left(\mathcal{E}^{\mu \nu}\right)^{(1)}\right)=0
$$

and the partially conserved current is $\mathcal{J}^{\nu}=\sqrt{-\bar{g}} \bar{\xi}_{\mu}\left(\mathcal{E}^{\mu \nu}\right)^{(1)}$. Hence, we must compute ${ }^{2}$
$\bar{\xi}_{\mu}\left(\mathcal{E}^{\mu \nu}\right)^{(1)}=\frac{1}{\kappa} \bar{\xi}_{\mu}\left(\mathcal{G}^{\mu \nu}\right)^{(1)}-\frac{\Lambda_{0}-\Lambda}{\kappa} \bar{\xi}_{\mu} h^{\mu \nu}+\sigma \bar{\xi}_{\mu}\left(E^{\mu \nu}\right)^{(1)}$.

We have already computed the first part in the previous subsection, and hence, the new parts are the second and the third terms. But when the theory is not given, one cannot proceed further from this point. For this reason, let us consider the quadratic theory as an example which also covers all the $f($ Riemann $)$ type theories. The action of the quadratic theory is

$$
\begin{aligned}
I= & \int d^{n} x \sqrt{-g}\left(\frac{1}{\kappa}\left(R-2 \Lambda_{0}\right)+\alpha R^{2}+\beta R_{\mu \nu} R^{\mu \nu}\right. \\
& \left.+\gamma\left(R_{\mu \nu \rho \sigma} R^{\mu \nu \rho \sigma}-4 R_{\mu \nu} R^{\mu \nu}+R^{2}\right)\right),
\end{aligned}
$$

and the field equations are [4]

$$
\begin{aligned}
& \frac{1}{\kappa}\left(R_{\mu \nu}-\frac{1}{2} g_{\mu \nu} R+g_{\mu \nu} \Lambda_{0}\right)+2 \alpha R\left(R_{\mu \nu}-\frac{1}{4} g_{\mu \nu} R\right)+(2 \alpha+\beta)\left(g_{\mu \nu} \square-\nabla_{\mu} \nabla_{\nu}\right) R \\
& \quad+2 \gamma\left(R R_{\mu \nu}-2 R_{\mu \sigma \nu \rho} R^{\sigma \rho}+R_{\mu \sigma \rho \tau} R_{\nu}{ }^{\sigma \rho \tau}-2 R_{\mu \sigma} R_{\nu}^{\sigma}-\frac{1}{4} g_{\mu \nu}\left(R_{\alpha \beta \rho \sigma} R^{\alpha \beta \rho \sigma}-4 R_{\alpha \beta} R^{\alpha \beta}+R^{2}\right)\right) \\
& \quad+\beta \square\left(R_{\mu \nu}-\frac{1}{2} g_{\mu \nu} R\right)+2 \beta\left(R_{\mu \sigma \nu \rho}-\frac{1}{4} g_{\mu \nu} R_{\sigma \rho}\right) R^{\sigma \rho}=\tau_{\mu \nu} .
\end{aligned}
$$

Inserting (13) in the last equation, one finds the equation satisfied by $\Lambda$ :

$\left.\frac{\Lambda-\Lambda_{0}}{2 \kappa}+\left((n \alpha+\beta) \frac{(n-4)}{(n-2)^{2}}+\gamma \frac{(n-3)(n-4)}{(n-1)(n-2)}\right) \Lambda^{2}\right)=0$.

Defining the constant

$c:=\frac{1}{\kappa}+\frac{4 \Lambda n}{n-2} \alpha+\frac{4 \Lambda}{n-1} \beta+\frac{4 \Lambda(n-3)(n-4)}{(n-1)(n-2)} \gamma$,

one can show that the linearized expressions read

\footnotetext{
${ }^{2}$ At this stage, we can search for a modified version of the $\mathcal{P}$ tensor, which is conserved and the trace of which is the $\mathcal{E}$-tensor. One can find such a tensor, but it does not have the symmetries of the Riemann tensor anymore, and hence it does not make the ensuing computation any simpler.
}

$$
\begin{aligned}
& \sigma \bar{\xi}_{\nu}\left(E^{\mu \nu}\right)^{(1)}-\frac{\Lambda_{0}-\Lambda}{\kappa} \bar{\xi}_{\nu} h^{\mu \nu} \\
& =\left(c-\frac{1}{\kappa}+\frac{4 \Lambda}{(n-1)(n-2)} \beta\right) \bar{\xi}_{\nu}\left(\mathcal{G}^{\mu \nu}\right)^{(1)} \\
& \quad+2 \beta \bar{\nabla}_{\alpha}\left(\bar{\xi}_{\nu} \bar{\nabla}^{[\alpha}\left(\mathcal{G}^{\mu] \nu}\right)^{(1)}+\left(\mathcal{G}^{\nu[\alpha}\right)^{(1)} \bar{\nabla}^{\mu]} \bar{\xi}_{\nu}\right) \\
& \quad+(2 \alpha+\beta) \bar{\nabla}_{\alpha}\left(2 \bar{\xi}^{\mu \mu} \bar{\nabla}^{\alpha]}(R)^{(1)}+(R)^{(1)} \bar{\nabla}^{\mu} \bar{\xi}^{\alpha}\right),
\end{aligned}
$$

which then yields the desired result

$$
\begin{aligned}
\bar{\xi}_{\nu}\left(\mathcal{E}^{\mu \nu}\right)^{(1)}= & \left(c+\frac{4 \Lambda}{(n-1)(n-2)} \beta\right) \bar{\xi}_{\nu}\left(\mathcal{G}^{\mu \nu}\right)^{(1)} \\
& +2 \beta \bar{\nabla}_{\alpha}\left(\bar{\xi}_{\nu} \bar{\nabla}^{[\alpha}\left(\mathcal{G}^{\mu] \nu}\right)^{(1)}+\left(\mathcal{G}^{\nu[\alpha}\right)^{(1)} \bar{\nabla}^{\mu]} \bar{\xi}_{\nu}\right) \\
& +(2 \alpha+\beta) \bar{\nabla}_{\alpha}\left(2 \bar{\xi}^{\mu \mu} \bar{\nabla}^{\alpha]}(R)^{(1)}+(R)^{(1)} \bar{\nabla}^{\mu} \bar{\xi}^{\alpha}\right) .
\end{aligned}
$$

Therefore, the conserved charges in quadratic gravity in $(\mathrm{A}) \mathrm{dS}$ read as 


$$
Q(\bar{\xi})=\frac{(n-1)(n-2)}{4 \Lambda(n-3)}\left(c+\frac{4 \Lambda \beta}{(n-1)(n-2)}\right) \int_{\partial \bar{\Sigma}} d^{n-2} x \sqrt{\bar{\gamma}} \bar{\epsilon}_{\mu \nu}\left(R_{\beta \sigma}^{\nu \mu}\right)^{(1)} \overline{\mathcal{F}} \beta \sigma .
$$

Observe that for asymptotically AdS spacetimes, the second and third lines in (35) do not contribute. But if one tries to generalize the above procedure to asymptotically nonconstant curvature spacetimes, those parts will also contribute generically. Therefore, for asymptotically AdS spacetimes, the only difference between the conserved charges in Einstein's theory (20) and the quadratic theory is the numerical factor in (36). Using the ideas presented in Ref. [10], the above construction can be extended to any $f$ (Riemann) theory, where $f$ is a smooth function. This is because, as far as the energy, vacua, and particle contents are considered, any $f$ (Riemann) theory has an equivalent quadratic action formulation in which one computes only three quantities $f\left(\bar{R}_{\alpha \beta}^{\mu \nu}\right), \frac{\partial f}{\partial R_{\alpha \beta}^{\mu \nu}}, \frac{\partial^{2} f}{\partial R_{\alpha \beta}^{\mu \nu} R_{\eta \delta}^{p \sigma}}$ and their contractions to find the $\kappa, \alpha, \beta$, and $\gamma$ of the theory to insert in (36). As this issue is dealt with in Refs. [10,11], we refer the reader to these works. So, the crucial part is the Einsteinian part, which we have studied in the previous section.

\section{GAUGE-INVARIANCE ISSUE}

The problem of the gauge transformations of the charge and the current that yields the charge is important. Clearly, one expects the charge to be gauge invariant in any valid formulation, but the current need not be. In fact, earlier constructions of conserved charges [4,5] used gaugevariant currents which yielded gauge-invariant charges. Of course, for the charges to be gauge invariant, the noninvariance of the current is only up to a boundary term that vanishes in the boundary. Let us show this in the expression of Ref. [4] for the cosmological Einstein theory,

$$
2 \bar{\xi}_{\nu}\left(\mathcal{G}^{\mu \nu}\right)^{(1)}=\bar{\nabla}_{\alpha} \mathcal{J}^{\alpha \mu},
$$

where the antisymmetric current is

$$
\begin{aligned}
\mathcal{J}^{\alpha \mu}:= & \bar{\xi}^{\alpha} \bar{\nabla}_{\beta} h^{\mu \beta}-\bar{\xi}^{\mu} \bar{\nabla}_{\beta} h^{\alpha \beta}+\bar{\xi}_{\nu} \bar{\nabla}^{\mu} h^{\alpha \nu} \\
& -\bar{\xi}_{\nu} \bar{\nabla}^{\alpha} h^{\mu \nu}+\bar{\xi}^{\mu} \bar{\nabla}^{\alpha} h-\bar{\xi}^{\alpha} \bar{\nabla}^{\mu} h \\
& +h^{\mu \nu} \bar{\nabla}^{\alpha} \bar{\xi}_{\nu}-h^{\alpha \nu} \bar{\nabla}^{\mu} \bar{\xi}_{\nu}-h \bar{\nabla}^{\alpha} \bar{\xi}^{\mu} .
\end{aligned}
$$

Consider an infinitesimal coordinate transformation generated by a vector field $\zeta$ (not to be confused with the Killing field $\xi$ ); one has

$$
\delta_{\zeta} h_{\mu \nu}=\bar{\nabla}_{\mu} \zeta_{\nu}+\bar{\nabla}_{\nu} \zeta_{\mu}=\mathcal{L}_{\zeta} \bar{g}_{\mu \nu},
$$

where $\mathcal{L}_{\zeta}$ denotes the Lie derivative and hence $\delta_{\zeta} h^{\mu \nu}=$ $-\mathcal{L}_{\zeta} \bar{g}^{\mu \nu}$. It is easy to see that $\delta_{\zeta}\left(\mathcal{G}^{\mu \nu}\right)^{(1)}=\mathcal{L}_{\zeta} \overline{\mathcal{G}}^{\mu \nu}=0$. But this only implies from (37) that one has the divergence of the gauge-transformed current to vanish

$$
\bar{\nabla}_{\alpha} \delta_{\zeta} \mathcal{J}^{\alpha \mu}=0,
$$

and hence $\mathcal{J}^{\alpha \mu}$ is not necessarily gauge invariant. In fact, one can show that $\mathcal{J}^{\alpha \mu}$ varies, under the gauge transformations (39), as

$$
\begin{aligned}
\delta_{\zeta} \mathcal{J}^{\alpha \mu}= & \bar{\nabla}_{\nu}\left(\bar{\xi}^{\alpha} \bar{\nabla}^{\nu} \zeta^{\mu}+\bar{\xi}^{\mu} \bar{\nabla}^{\alpha} \zeta^{\nu}+\bar{\xi}^{\nu} \bar{\nabla}^{\mu} \zeta^{\alpha}+2 \zeta^{\alpha} \bar{\nabla}^{\nu} \bar{\xi}^{\mu}\right. \\
& \left.+\zeta^{\nu} \bar{\nabla}^{\mu} \bar{\xi}^{\alpha}-(\mu \leftrightarrow \alpha)\right) .
\end{aligned}
$$

Clearly, since the variation is a boundary term and since $\mathcal{J}^{\alpha \mu}$ is the integrand on the boundary of the spatial slice, the boundary term does not contribute to the charges (as $\partial \partial \bar{\Sigma}=0$ ), and hence the charge is gauge invariant. But this exercise shows us that the current (38) is only gauge invariant up to a boundary term.

On the other hand, since $\delta_{\zeta}\left(R_{\beta \sigma}^{\nu \mu}\right)^{(1)}$ is gauge invariant, our charge expression (20) is explicitly gauge invariant without an additional boundary term. Let us show this:

$$
\delta_{\zeta}\left(R_{\beta \sigma}^{\nu \mu}\right)^{(1)}=\bar{g}^{\alpha \mu} \delta \zeta\left(R_{\alpha \beta \sigma}^{\nu}\right)^{(1)}-\bar{R}_{\alpha \beta \sigma}^{\nu} \delta_{\zeta} h^{\alpha \mu} .
$$

Given the linearized Riemann tensor as

$$
\left(R_{\alpha \beta \sigma}^{\nu}\right)^{(1)}=\bar{\nabla}_{\beta}\left(\Gamma_{\sigma \alpha}^{\nu}\right)^{(1)}-\bar{\nabla}_{\sigma}\left(\Gamma_{\beta \alpha}^{\nu}\right)^{(1)},
$$

one needs

$$
\delta_{\zeta}\left(\Gamma_{\sigma \alpha}^{\nu}\right)^{(1)}=\bar{\nabla}_{\sigma} \bar{\nabla}_{\alpha} \zeta^{\nu}+\bar{R}_{\alpha \rho \sigma}^{\nu} \zeta^{\rho} .
$$

Collecting all the pieces together, one arrives at

$$
\delta_{\zeta}\left(R_{\beta \sigma}^{\nu \mu}\right)^{(1)}=\mathcal{L}_{\zeta} \bar{R}_{\beta \sigma}^{\nu \mu} .
$$

For the AdS background, one clearly has $\mathcal{L}_{\zeta} \bar{R}^{\nu \mu}{ }_{\beta \sigma}=0$ and hence $\delta_{\zeta}\left(R_{\beta \sigma}^{\nu \mu}\right)^{(1)}=0$, and so $\delta_{\zeta} Q=0$ as expected. So, in our formalism, not only is the charge explicitly gauge invariant, but also the current is explicitly gauge invariant.

In addition to the above discussion of gauge invariance which amounts to changing the coordinates under which the field transforms as (39), one can consider transformations which are isometries of the background spacetime. Under the latter transformations, the $h_{\mu \nu}$ field transforms as a $(0,2)$ tensor field. In the case of AdS spacetime, these transformations form the group $O(D-1,2)$ for $D \geq 4$ and an infinite-dimensional group in $D=3$ dimensions [12]. As opposed to the "gauge symmetries" above, these are 
genuine symmetries of the background spacetime. Namely, the generators of these transformations are conserved nontrivial charges. Of course, the components of the background Killing vectors transform as vectors under these isometries, and in general, one gets a superposition of Killing vectors which is a Killing vector itself. So, as expected, the conserved charges (as generators of symmetries) satisfy the isometry algebra.

\section{SOME ASYMPTOTICALLY ADS SPACETIMES}

We have given the computation of the energy and the angular momentum of the four-dimensional Kerr-AdS solution in Ref. [3]; here, let us give two more examples.

\section{A. $\boldsymbol{n}$-dimensional AdS-Schwarzschild spacetime}

Consider a spherically symmetric metric in $n$ dimensions:

$$
\mathrm{d} s^{2}=-f(r) \mathrm{d} t^{2}+\frac{1}{f(r)} \mathrm{d} r^{2}+r^{2} \mathrm{~d} \Omega_{n-2} .
$$

For the choice

$$
f(r)=1-\left(\frac{r_{0}}{r}\right)^{n-3}+\frac{r^{2}}{\ell^{2}}, \quad \ell^{2} \equiv-\frac{(n-1)(n-2)}{2 \Lambda},
$$

the metric (46) is an Einstein spacetime with the Ricci tensor

$$
R_{\mu \nu}=-\frac{n-1}{\ell^{2}} g_{\mu \nu} .
$$

As such, it solves the $n$-dimensional vacuum Einstein equations with a cosmological constant. For the Killing vector $\xi^{\mu}=(-1,0, \ldots, 0)$, one needs to compute the integrand in (21), which boils down to computing the expression $\left(R_{r t}^{r t}\right)^{(1)} \bar{\nabla}^{r} \bar{\xi}^{t}$. It is easy to see that the relevant component of the full Riemann tensor reads

$R_{r t}^{r t}=-\frac{1}{2} f^{\prime \prime}(r), \quad\left(R_{r t}^{r t}\right)^{(1)}=-(n-3)(n-2) \frac{r_{0}^{n-3}}{r^{n-2}}$,

where $f^{\prime}(r)=\frac{d f(r)}{d r}$. Similarly, one has $\bar{\nabla}^{r} \bar{\xi}^{t}=-\left.\frac{f^{\prime}}{2}\right|_{r_{0} \rightarrow 0}=$ $-\frac{r}{\ell^{2}}$. Combining all these in (21) for $G=1$, one finds the energy of (46) as

$$
E=\frac{n-2}{4} r_{0}^{n-3},
$$

which is exactly the one computed in Ref. [4]. In four dimensions, one has $r_{0}=2 m$ and $E=m$.

\section{B. AdS soliton}

The metric of the "AdS soliton" was found by Horowitz and Myers [13] and reads as

$$
\begin{aligned}
d s^{2}= & \frac{r^{2}}{\ell^{2}}\left[\left(1-\frac{r_{0}^{p+1}}{r^{p+1}}\right) d \tau^{2}+\sum_{i=1}^{p-1}\left(d x^{i}\right)^{2}-d t^{2}\right] \\
& +\left(1-\frac{r_{0}^{p+1}}{r^{p+1}}\right)^{-1} \frac{\ell^{2}}{r^{2}} d r^{2}
\end{aligned}
$$

We shall not go into the physical meaning of this solution, which is obtained from a $p$-brane metric; the Cartesian coordinates $x^{i}(i=1, \ldots, p-1)$ and the $t$ denote the coordinates on the "brane" and $r \geq r_{0}$. The solution does not have a singularity if the coordinate $\tau$ is periodic with a period $\beta=4 \pi \ell^{2} /\left(r_{0}(p+1)\right)$. Consider the timelike Killing vector

$$
\bar{\xi}^{\mu}=(-1,0, \ldots, 0)
$$

then, $\bar{\nabla}^{r} \bar{\xi}^{t}=\frac{-r}{e^{2}}$. The relevant linearized Riemann tensor component can be computed to be

$$
\left(R_{r t}^{r t}\right)^{(1)}=-\frac{(n-3)}{2 \ell^{2}} \frac{r_{0}^{n-1}}{r^{n-1}},
$$

which also shows that there is no $n=3$ AdS soliton. Making use of (21), one obtains

$$
E=-\frac{V_{n-3} \pi}{(n-1) \Omega_{n-2}} \frac{r_{0}^{n-2}}{\ell^{n-2}},
$$

where $V_{n-3}$ is the volume of the compact dimensions. This result matches the one obtained in Ref. [14].

\section{CONCLUSIONS}

In a gauge or gravity theory, the conserved charges make sense if they are gauge or coordinate invariant (at least for small transformations). The ADM [6] and AD [5] charges and their generalizations to higher order gravity [4] are all gauge invariant. Namely, they are invariant under small diffeomorphisms. (Large diffeomorphisms are a different story; even the flat Minkowski space, while remaining flat, can be assigned any mass value in a coordinate system that does not have proper asymptotics. See Ref. [7] for a brief review of this issue.) However, the explicit expressions of these charges do not involve the relevant gauge-invariant quantity, that is, the linearized Riemann tensor with two up and two down indices, $\left(R^{\mu \nu}{ }_{\sigma \rho}\right)^{(1)}$, but instead they involve the first covariant derivative of the metric perturbation as $\bar{\nabla}_{\alpha} h_{\mu \nu}$ contracted with the Killing vector in such a way that the final result is gauge invariant only up to a divergence term which vanishes in the boundary. The obvious question is to try to understand if the gauge-invariant charges can be 
written in an explicitly gauge-invariant way with the help of the Riemann tensor.

There is a stronger motivation for such a search: outside the sources, the Riemann tensor carries all the information about gravity. Naturally, it must carry the information about the conserved charges. It turns out, as we have shown recently [3] and here, this is indeed the case and the conserved charge is basically a flux of the Riemann tensor at spatial infinity contracted with an antisymmetric 2tensor. The construction is somewhat nontrivial and is valid only for asymptotically AdS spacetimes (which can be generalized to Einstein spacetimes with a nonzero scalar curvature). More specifically, in the cosmological Einstein theory, our construction remains intact for any Einstein spacetimes as long as there is a background Killing vector field. For generic gravity theories, the construction is analogous, but there arise many more terms in the final expressions. For an example of this, see the quadratic gravity studied in Ref. [15].

The reason that one can write the conserved charges as a flux of the linearized Riemann tensor at all is that for AdS spacetimes a given Killing vector $\bar{\xi}^{\mu}$ has an antisymmetric 2-form potential as $\bar{\xi}^{\mu}=\bar{\nabla}_{\nu} \overline{\mathcal{F}}^{\mu \nu}$, which helps bring another covariant derivative in the conserved charges whenever the Killing vector appears, converting the expression to the linearized Riemann tensor that has two covariant derivatives of the metric perturbation. To find the charge expression, we used a divergence-free rank-4 tensor of which the trace is the Einstein tensor. Interestingly, this construction is valid only for $n \geq 4$ dimensions and is not valid in three dimensions, since the Riemann tensor can be expressed directly in terms of the Einstein tensor in three dimensions, the linearized version of which vanishes at spatial infinity.

Finally, we should note that it was realized a long time ago by Regge and Teitelboim [16] that in the fully nonlinear Hamiltonian treatment of general relativity in spatially open manifolds one has to include a boundary term $E[g]$ to the bulk Hamiltonian for the functional derivatives of the functionals with respect to the canonical fields to make sense. Namely, to reproduce Einstein's equations from the Hamiltonian equations, one must add a surface term to the Hamiltonian, which, on the appearance, does not modify the Hamiltonian equations but makes them well defined. That term turns out to be the ADM energy $\left(E[g]=E_{\mathrm{ADM}}[h]\right)$ given by the derivative of the linearized metric at spatial infinity. Moreover, the value of the full Hamiltonian, say $H$, which is a sum of the bulk and boundary terms, yields $H=E_{\mathrm{ADM}}[h]$ upon use of the field equations. Namely, the apparently linear-looking ADM energy captures all the nonlinear energy stored in the gravitational field and the localized matter in the bulk of the spacetime. The same construction works for the AbbottDeser energy in asymptotically AdS spacetimes, and here, we have given an explicitly gauge-invariant formulation of this energy and other conserved charges.
[1] H. Lu and C. N. Pope, Critical Gravity in Four Dimensions, Phys. Rev. Lett. 106, 181302 (2011).

[2] S. Deser, H. Liu, H. Lu, C. N. Pope, T. C. Sisman, and B. Tekin, Critical points of $D$-dimensional extended gravities, Phys. Rev. D 83, 061502 (2011).

[3] E. Altas and B. Tekin, Conserved charges in AdS : A new formula, arXiv:1811.00370.

[4] S. Deser and B. Tekin, Energy in generic higher curvature gravity theories, Phys. Rev. D 67, 084009 (2003); Gravitational Energy in Quadratic Curvature Gravities, Phys. Rev. Lett. 89, 101101 (2002).

[5] L. F. Abbott and S. Deser, Stability of gravity with a cosmological constant, Nucl. Phys. B195, 76 (1982).

[6] R. Arnowitt, S. Deser, and C. W. Misner, Canonical variables for general relativity, Phys. Rev. 117, 1595 (1960); The dynamics of general relativity, Gen. Relativ. Gravit. 40, 1997 (2008).

[7] H. Adami, M. R. Setare, T. C. Sisman, and B. Tekin, Conserved charges in extended theories of gravity, arXiv:1710.07252.

[8] A. Petrov, S. Kopeikin, R. Lompay, and B. Tekin, Metric Theories of Gravity, Perturbations and Conservation Laws (De Gruyter, Berlin, 2017).
[9] G. Compere and A. Fiorucci, Advanced lectures in general relativity, arXiv:1801.07064.

[10] C. Senturk, T. C. Sisman, and B. Tekin, Energy and angular momentum in generic $\mathrm{f}$ (Riemann) theories, Phys. Rev. D 86, 124030 (2012).

[11] A. J. Amsel and D. Gorbonos, Wald-like formula for energy, Phys. Rev. D 87, 024032 (2013).

[12] J.D. Brown and M. Henneaux, Central charges in the canonical realization of asymptotic symmetries: An example from three-dimensional gravity, Commun. Math. Phys. 104, 207 (1986).

[13] G. T. Horowitz and R. C. Myers, The AdS/CFT correspondence and a new positive energy conjecture for general relativity, Phys. Rev. D 59, 026005 (1998).

[14] H. Cebeci, O. Sarioglu, and B. Tekin, Negative mass solitons in gravity, Phys. Rev. D 73, 064020 (2006).

[15] D. O. Devecioglu and O. Sarioglu, Conserved Killing charges of quadratic curvature gravity theories in arbitrary backgrounds, Phys. Rev. D 83, 021503 (2011).

[16] T. Regge and C. Teitelboim, Role of surface integrals in the Hamiltonian formulation of general relativity, Ann. Phys. (N.Y.) 88, 286 (1974). 